\title{
Role of gastrointestinal endoscopy in the screening of digestive tract cancers in Europe: European Society of Gastrointestinal Endoscopy (ESGE) Position Statement
}

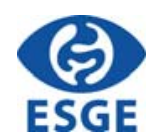

Authors

Adrian Săftoiu' ${ }^{1,2}$, Cesare Hassan ${ }^{3}$, Miguel Areia4,5, Manoop S. Bhutani ${ }^{6}$, Raf Bisschops ${ }^{7}$, Erwan Bories ${ }^{8}$, Irina M. Cazacu $^{1,6}$, Evelien Dekker ${ }^{9}$, Pierre H. Deprez ${ }^{10}$, Stephen P. Pereira ${ }^{11}$, Carlo Senore ${ }^{12}$, Riccardo Capocaccia ${ }^{13}$, Giulio Antonelli ${ }^{3}$, Jeanin van Hooft ${ }^{9}$, Helmut Messmann ${ }^{14}$, Peter D. Siersema ${ }^{15}$, Mario Dinis-Ribeiro ${ }^{5,16}$, Thierry Ponchon ${ }^{17}$

Institutions

1 Research Center of Gastroenterology and Hepatology Craiova, University of Medicine and Pharmacy, Craiova, Romania

2 Gastroenterology Department, Regina Maria-Ponderas Academic Hospital, Bucharest, Romania

3 Nuovo Regina Margherita Hospital, Rome, Italy

4 Gastroenterology Department, Portuguese Oncology Institute of Coimbra, Portugal

5 Center for Research in Health Technologies and Information Systems (CINTESIS), Faculty of Medicine, Porto, Portugal

6 Department of Gastroenterology, Hepatology and Nutrition, MD Anderson Cancer Center, The University of Texas, Houston, USA

7 Department of Gastroenterology and Hepatology, Catholic University of Leuven (KUL), TARGID, University Hospitals Leuven, Leuven, Belgium

8 Private office, Aix-en-Provence, France

9 Department of Gastroenterology and Hepatology, Cancer Center Amsterdam, Amsterdam University Medical Centers, The Netherlands

10 Department of Hepato-Gastroenterology, Cliniques universitaires Saint-Luc, Université Catholique de Louvain, Brussels, Belgium

11 Institute for Liver and Digestive Health, Royal Free Hospital Campus, University College London, UK

12 Epidemiology and Screening Unit-CPO, University Hospital Città della Salute e della Scienza, Turin, Italy

13 Editorial Board, Epidemiologia e Prevenzione

14 III Med Klinik, Klinikum Augsburg, Augsburg, Germany

15 Department of Gastroenterology and Hepatology, Radboud University Medical Center, Nijmegen, The Netherlands

16 Gastroenterology Department, Portuguese Oncology Institute of Porto, Portugal

17 Gastroenterology Division, Edouard Herriot Hospital, Lyon, France
Bibliography

DOI https://doi.org/10.1055/a-1104-5245

Published online: 12.2.2020 | Endoscopy 2020; 52: 293-304

(c) Georg Thieme Verlag KG Stuttgart · New York

ISSN 0013-726X

Corresponding author

Adrian Saftoiu, MD PhD, Research Center of

Gastroenterology and Hepatology, University of Medicine

and Pharmacy, Craiova, Romania

Fax: $+40-251-310287$

adrian.saftoiu@umfcv.ro

adriansaftoiu@gmail.com

\section{SUMMARY OF STATEMENTS}

In Europe at present, but also in 2040, 1 in 3 cancer-related deaths are expected to be caused by digestive cancers. Endoscopic technologies enable diagnosis, with relatively low invasiveness, of precancerous conditions and early cancers, thereby improving patient survival. Overall, endoscopy capacity must be adjusted to facilitate both effective screening programs and rigorous control of the quality assurance and surveillance systems required.

1 For average-risk populations, ESGE recommends the implementation of organized population-based screening programs for colorectal cancer, based on fecal immunochemical testing (FIT), targeting individuals, irrespective of gender, aged between 50 and 75 years. Depending on local factors, namely the adherence of the target population and availability of endoscopy services, primary screening by colonoscopy or sigmoidoscopy may also be recommendable.

2 In high-risk populations, endoscopic screening for gastric cancer should be considered for individuals aged more than 40 years. Its use in countries/regions with intermediate risk may be considered on the basis of local settings and availability of endoscopic resources. 
3 For esophageal and pancreatic cancer, endoscopic screening may be considered only in high-risk individuals:

- For squamous cell carcinoma, in those with a personal history of head/neck cancer, achalasia, or previous caustic injury;

- For Barrett's esophagus (BE)-associated adenocarcinoma, in those with long-standing gastroesophageal reflux disease symptoms (i.e., $>5$ years) and multiple risk factors (age $\geq 50$ years, white race, male sex, obesity, first-degree relative with $B E$ or esophageal adenocarcinoma [EAC]).

- For pancreatic cancer screening, endoscopic ultrasound may be used in selected high-risk patients such as those with a strong family history and/or genetic susceptibility.

\section{SOURCE AND SCOPE}

This Guideline is an official statement of the European Society of Gastrointestinal Endoscopy (ESGE), reviewing the role of gastrointestinal (GI) endoscopy in the early diagnosis and prevention of $\mathrm{Gl}$ cancers.

\section{Introduction}

Mostly because of the aging population and environmental risk factors, gastrointestinal (GI) cancers represent a significant burden for European citizens, comprising one quarter of all the malignancies diagnosed in Europe. An estimated 600000 cases of $\mathrm{Gl}$ cancer and 360000 related deaths per year occur in the European Union [1]. Moreover, when diagnosed in a symptomatic phase, most $\mathrm{Gl}$ cancers are still associated with a dismal prognosis. The 5-year survival, as estimated for 2000-2007, was $41 \%$ overall, varying according to site of diagnosis: $12 \%$ for esophagus, $24 \%$ for stomach, $48 \%$ for colorectum, and $6 \%$ for pancreas [1]. In addition, late-stage cancers represent an economic and financial burden because of palliative treatment and new biological treatments for advanced disease.

Better understanding of the natural history of $\mathrm{GI}$ cancers has shown that most of them are preceded by slowly progressing precancerous conditions or lesions, as well as by early invasive stages, therefore providing opportunities for effective interventions. Beyond the classic adenoma-carcinoma sequence for colorectal carcinogenesis, similar pathways based on metaplasia-dysplasia-cancer progression have been shown for upper $\mathrm{Gl}$ as well as pancreatic cancers. In addition, advances in knowledge about genetic factors have led to the identification of the pathogenetic mutations responsible for familial $\mathrm{GI}$ cancer syndromes.

In this area, the application of endoscopic techniques to the Gl tract, i.e., gastrointestinal endoscopy, represents a set of unique technologies for early detection of cancer or precursors, followed by potential endoscopic interventions. In fact, endoscopic resection of precancerous and early lesions has been associated with reduction of incidence and very high 5-year survival rates. Besides, it is usually preferred by patients because it is less invasive and less costly than surgical treatments. Moreover, endoscopic and histological diagnosis of precancerous conditions leads to an effective risk stratification of the endos- copy population, with appropriate adjustment of surveillance protocols to those high-risk patients who may benefit the most.

In this document, ESGE aims to summarize its position regarding the current role of endoscopy in screening for the diverse gastrointestinal neoplasms, and to support the role of digestive endoscopy in the reduction of cancer incidence and mortality. Discussion of the organization of screening programs lies outside the scope of this Position Statement, whilst alternatives to endoscopy will be briefly considered. In fact, despite the substantial developments in our own field and significant relevant evidence for the impact of endoscopy, as briefly stated above, lack of awareness by other stakeholders may lead to underuse or poor resourcing of health facilities involved in providing screening services, with consequent failure to fully realize the potential benefits to patients.

\section{Methods}

In 2017, the European Society of Gastrointestinal Endoscopy (ESGE) Governing Board established a task force (Public Affairs Working Group led by A.S.) to produce a Position Statement concerning the value of endoscopy for screening purposes in Gl cancers. The most prevalent digestive cancers (esophageal squamous cell carcinoma, esophageal adenocarcinoma, gastric carcinoma, colorectal cancer, and pancreatic cancer) were considered.

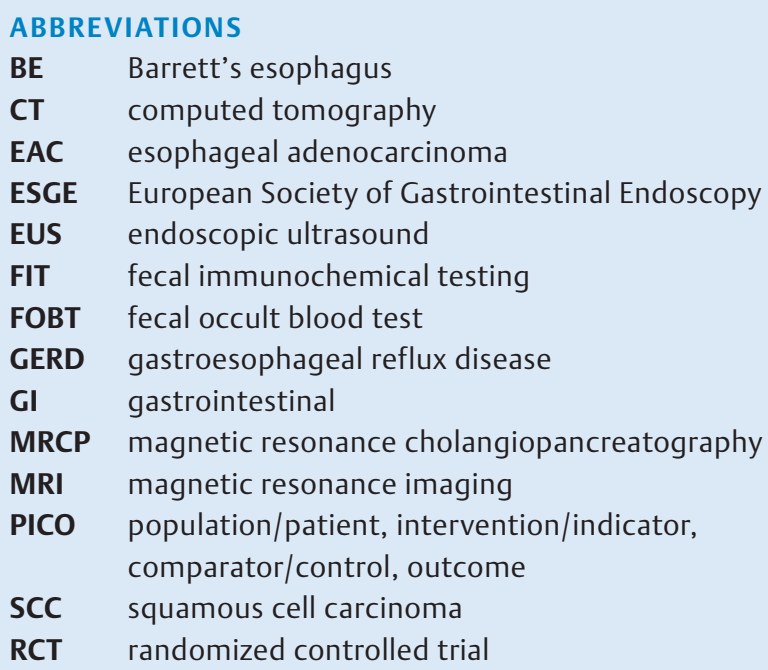


Using a structured PICO framework (population/patient, intervention/indicator, comparator/control, outcome), detailed literature searches were performed by an expert task force, yielding results, through a modified Delphi process, that are summarized in recommendations/statements. The PICO items were defined regarding the role, in terms of prevention, survival, and cost-effectiveness (outcomes) of digestive endoscopy technologies (upper gastrointestinal endoscopy, colonoscopy, and endoscopic ultrasound [EUS]; interventions and comparators) in two different settings (populations/patients), namely for the average-risk population and for high-risk groups/settings defined by geography, ethnicity, individual exposure, or family history. This excludes the specific management of individuals with known hereditary colorectal cancer syndromes or with known precancerous gastric lesions, as considered in previous ESGE Guidelines [2,3].

Epidemiological data were taken from online databases available from international studies. Pooled national estimates have been used, derived from European incidence and mortality data in 2018 provided by the Joint Research Center and European Network of cancer registries [4]. Incidence time trends in 2004-2010 are based on observed rates from a pool of population-based cancer registries and are available from the same database. Histology-specific data for esophageal cancer were available from the RARECARENet database on incidence and survival for rare tumors in Europe [5]. Survival data were downloaded from the EUROCARE-5 project database [1].

\section{Role of digestive endoscopy for average-risk populations: colon cancer}

\section{STATEMENT}

For colorectal cancer, ESGE recommends the implementation of organized population-based screening programs for average-risk populations based on fecal immunochemical testing (FIT), targeting individuals, irrespective of gender, aged between 50 and 75 years. Depending on local factors, namely the adherence of the target population and availability of endoscopy services, primary screening by colonoscopy or sigmoidoscopy may also be recommendable.

\section{Frequency and pathogenesis: colorectal cancer}

Colorectal cancer (CRC) represents the second commonest cause of cancer morbidity and mortality in Europe, with an estimated 380000 new cases and 175000 related deaths in 2018 [6]. CRC is uniquely suited for screening programs compared with other $\mathrm{Gl}$ cancers as it has detectable precursor lesions (adenomatous polyps) resection of which decreases the incidence and prevalence of CRC, with a consequent reduction in mortality.

\section{Target population: CRC screening}

Most screening programs include the general population aged 50 to 75 years. The range may vary according to availability of resources. In the US, because of increased incidence of CRC in young adults, the target group was recently expanded to include individuals aged 45 and older. The same trend in incidence of CRC has been seen in Europe over the last 25 years [7].

\section{Role of endoscopy}

Screening of average-risk individuals by fecal occult blood testing (FOBT) followed by colonoscopy for positive cases, and by primary lower GI endoscopy (either colonoscopy or sigmoidoscopy) can significantly reduce CRC incidence and/or related mortality $[8,9]$. This is the result of two effects, namely downstaging of existing CRC by early diagnosis, and prevention of the development of CRC by removal of precancerous polyps.

For guaiac FOBT, the evidence is provided by four RCTs that showed an overall $24 \%$ reduction in CRC mortality among those undergoing screening [9]. As FIT has a 2-3-fold higher sensitivity for detecting advanced neoplasia than the guaiac-based test, a greater effect of FIT-based screening is expected [10]. The efficacy of primary sigmoidoscopy screening is supported by two randomized controlled trials (RCTs) showing an overall $21 \%$ and $28 \%$ reduction in CRC incidence and mortality, respectively, for those offered screening. The protective effects for those actually undergoing screening were $31 \%$ and $33 \%$ for incidence and $38 \%$ and $43 \%$ for mortality $[11,12]$. However, participation rates are much lower than for FIT.

The efficacy of colonoscopy screening is long-lasting [13] and the test may even be performed only once in a lifetime $[14,15]$. However, the long-term efficacy in preventing CRC has been associated with the quality of the screening, as reported in the ESGE document on this subject [16]. Only observational studies are available for the assessment of colonoscopy screening, and these estimate reductions in CRC incidence and mortality of $69 \%$ and $68 \%$, respectively [8]. Moreover, the population coverage with regard to opportunistic CRC screening remains disappointingly low. In 2017, it was estimated that only a small minority $(0.4 \%-4 \%)$ of the European population had undergone a colonoscopy in the previous 10 years, as compared with over $60 \%$ in the United States [17]. This is probably related to multifactorial barriers, including personal beliefs (e. g. lack of awareness of or fear of the screening test), organizational issues (e.g. lack of recommendation by primary care physicians), and financial barriers [18].

In Europe, according to the EU screening report, most countries have in fact implemented organized invitational screening programs based on FIT [19]. The main advantages of organized versus opportunistic screening are the active invitation of all eligible individuals and the implementation of quality assurance programs. In addition, there is the potential for sending reminders to increase participation and for proactive removal of any organizational barriers to patients' navigation throughout the screening process. Compared with nonorganized settings, organized programs have also been shown to result in high compliance with follow-up of those with a positive 
primary screening finding, and high adherence in subsequent screening rounds of those with a negative test result [20]. Organized screening programs, however, require several resources. Two of the most challenging aspects of an organized program are the heavy burden on the available endoscopic capacity and the high costs. The most reasonable solution for the first problem would be to proportionally increase the number of endoscopists in order to match the additional burden of colonoscopies for the screening program, including for surveillance thereafter $[21,22]$. In addition, to prevent unnecessary extra colonoscopies due to inappropriate indications, surveillance guidelines should be strictly followed [23].

As mentioned above, organized programs require complex organizational activities that, considering the large variability in the structure of the different health systems, are not necessarily available in all European countries. Indeed, some regions or countries in the EU have not yet implemented screening programs [24]. In these countries, average-risk patients may exceptionally apply for a case-by-case or 'opportunistic' nonorganized screening for CRC prevention.

Although CRC screening may have been implemented, the actual coverage of the target population by invitation has shown a wide variation across the EU member states, ranging between $1.5 \%$ and $100 \%$. Equally, participation rates vary widely across EU countries, resulting in an actual screening coverage of $19.8 \%$ for the entire $50-74$ target age range in population-based programs, and $25.1 \%$ in the age ranges targeted by the programs [25]. Although this is an underestimate (as several programs could not provide adequate data about opportunistic screening activities), the corresponding figure for non-population-based programs was as low as $4.2 \%$ [8].

\section{Cost-effectiveness of CRC screening}

The convenience of population-based CRC screening has been shown in several simulation models. In particular, such screening has been demonstrated to be cost-saving, or cost-neutral, because of the substantial decrease in expenditure on CRC treatment, including biological therapy, that has been achieved by reduction in CRC incidence and by downstaging of existing cancers [26].

Surveillance for individuals at increased risk of CRC because of personal or family CRC history has been addressed in previous ESGE Guidelines [2, 3].

\section{Role of digestive endoscopy for high- and intermediate-risk populations: gastric cancer}

\section{STATEMENT}

In high-risk populations, endoscopic screening for gastric cancer should be considered for individuals aged more than 40 years. Its use in countries/regions with intermediate risk may be considered on the basis of local settings and availability of endoscopic resources.

\section{Epidemiology: gastric cancer}

Gastric cancer is the fifth most common malignancy and the third leading cause of cancer death worldwide [27]. Gastric cancer incidence is decreasing in developed countries, but it is still responsible for about 80000 new cases per year in the EU, with an incidence of 16 per 100000 per year. Even though gastric cancer can be recognized and treated at an early point, most of these cancers are still diagnosed late, with an overall 5-year survival of $24 \%$. Nevertheless, mortality might be reduced by $40 \%$, with early detection by means of screening $[28,29]$.

Gastric cancer screening is intended for the intestinal type of gastric cancer, that represents more than $95 \%$ of all gastric cancers and is the final stage of the inflammation-metaplasiadysplasia-carcinoma sequence known as the Correa cascade [29]. The diffuse type of gastric cancer has a different carcinogenetic sequence and screening is not indicated. Currently, screening for intestinal-type gastric cancer is only performed in countries with a high disease incidence, defined as an agestandardized rate $\geq 20$ per 100000 , such as Japan or South Korea (29.9 and 41.3 per 100000 , respectively) [30,31]. Screening enables detection of gastric cancer at earlier stages, even of the early gastric cancers defined as carcinoma limited to the mucosa or submucosa, regardless of lymph node involvement; in other words lesions amenable to curative endoscopic treatment such as endoscopic submucosal dissection [32, 33].

\section{Target population: gastric cancer}

All the screening studies are from Asia and most have used the age range $40-80$ years since most gastric adenocarcinomas are diagnosed after the age of 40 years. This is similar to (although wider than) the 50-75-year range of the European colorectal cancer screening recommendation [28,34].

Patients with family history, pernicious anaemia, previous partial gastrectomy, or in other subgroups, lie outside the scope of the present statement and should follow specific recommendations.

\section{Role of endoscopy (upper Gl endoscopy)}

In high-risk areas (defined as having an age-standardized rate $\geq 20$ per 100000), endoscopy has a clear role for primary screening. The interval between examinations with negative findings varies among studies but most reported annual or biennial endoscopies [28]. Also in these regions, serologic screening based on pepsinogen testing has been promoted and its effectiveness demonstrated [34-36].

For regions with an age-standardized mortality rate for gastric cancer of $<10$ per 100000 , endoscopic screening for the entire population is not recommended [37].

For intermediate-risk regions, with an age-standardized rate between 10 and 20 per 100000 , endoscopy may have a role for primary screening if cost-effectiveness is proven in the particular country. The interval between negative exams might be every 5 years [38]. 


\section{Cost-effectiveness of screening for gastric cancer}

Several studies concluded that endoscopic screening is costeffective in high-incidence regions [39-43]. Indeed, the two most recent studies, both for the Korean population, concluded that endoscopic screening is cost-effective. Studies indicated that either annual screening for men and biennial for women, for a population aged $50-80$ years, or annual screening in patients older than 40 years is cost-effective [42-44].

In Europe, one study compared three screening strategies: stand-alone upper endoscopy; endoscopy combined with a CRC screening colonoscopy after a positive FOBT result; and pepsinogens serologic screening. It concluded that an endoscopic gastric cancer screening every 5 years was cost-effective only if combined with a screening colonoscopy [38]. This means that in Europe, if a colorectal cancer screening program is already in place (by means of FOBT or stand-alone colonoscopy) all countries with an intermediate incidence rate of gastric cancer, such as Albania, Belarus, Macedonia, Russia, Latvia, Ukraine, Estonia, Lithuania, Portugal, Moldova, Romania, Slovenia, Bulgaria, and Croatia (presented according to their agestandardized rate, from 20.1 to 10.3 ), might benefit by providing a screening upper endoscopy in conjunction with colonoscopy for their populations. Although prospective studies on the use of pepsinogen serology as a screening method are ongoing in some European countries, its high cost and limited availability outside Asia are the main limitations from a costeffectiveness and practical perspective; as such, it cannot be recommended at the moment $[3,35]$.

In the East, three other studies concluded that an endoscopic mass screening every 2 years was only cost-effective in high-risk individuals aged 50-70 years with an odds ratio for gastric cancer risk of $>3.9$, but not for the entire population $[43,45,46]$.

Only two studies in low-risk scenarios (USA) had been published, and these concluded that gastric cancer incidence would have to increase by $337 \%$ for screening to become costeffective $[47,48]$.

Surveillance for individuals at increased risk of gastric cancer because of a personal history of precancerous conditions or lesions has been addressed in previous ESGE Guidelines.

\section{Role of digestive endoscopy in high-risk settings: esophageal cancer}

\section{Squamous cell carcinoma (SCC)}

\section{STATEMENT}

Endoscopic screening for squamous cell carcinoma should only be considered in high-risk individuals or patients (opportunistic): i.e., in those with a personal history of head/neck cancer, achalasia, or previous caustic injury.

\section{Epidemiology: SCC}

Esophageal cancer is the seventh most commonly occurring cancer in men and 13th most common in women [49]. Globally, SCC accounts for the majority of the cases of esophageal cancer although its proportion relative to adenocarcinoma varies from country to country, being on average approximately $1: 1$ in the EU. In 2013 it was estimated that there were about 19200 new SCC diagnoses per year in the EU. Over the past three decades, a consistent decline in the rates of esophageal SCC has been observed in Western Europe and a stable rate or slower decline in central European countries. On the other hand, an increase in SCC incidence has been reported in Eastern European countries. The decline in SCC incidence in Western Europe has been mainly attributed to the reduction in alcohol consumption and smoking habits. SCC survival is low, being $38 \%$ at 1 year and $12 \%$ at 5 years after diagnosis [5].

\section{Target population: SCC}

In moderate- and lower-risk Western countries, the most important risk factors are the combination of tobacco smoking and excessive alcohol consumption [50]. Unlike adenocarcinoma, esophageal SCC is 3 to 5 times more likely among people who consume alcohol ( 3 or more drinks daily), and the risk increases synergistically with tobacco smoking. SCC screening in moderate- and lower-risk countries would include an excessively large population at risk and therefore seems impractical. Screening is therefore usually proposed to small subgroups of patients at very high risk, such as those with a previous or concomitant diagnosis of head/neck SCC [51], achalasia (up to 10 times risk) [52], previous radiotherapy for breast cancer, history of head/neck cancer, previous caustic injury to the esophagus, and tylosis [53].

There is no specific recommendation on the best time to start screening for SCC. For high-risk factors, achalasia is a good example of the absence of consensus, although the absolute risk increase for SCC was 308.1 per 100000 patients per year, suggesting a strict endoscopic surveillance for these patients. But in practice, no consensus has been reached among world experts with regard to timing: practices are still varied, with screening commencing at or within 1 year of diagnosis in some cases compared with 5 and 10 years in others. Surveillance intervals also vary, from 2 to 5 years [54].

\section{Role of endoscopy: SCC}

Precancerous dysplastic lesions are detectable using endoscopy and noninvasive screening methods; however routine screening is currently not recommended outside high-risk areas or for low-risk individuals [55]. Endoscopy remains the gold standard for diagnosis of dysplasia and early SCC but it is invasive and expensive, and therefore alternative approaches to broaden the test population are of interest. Since serologic tests are not clinically available yet, other invasive but less costly tests are needed to diagnose SCC or premalignant lesions, such as exfoliative cytology [56]. 


\section{Cost-effectiveness: SCC}

There are very few studies about the cost-effectiveness of SCC screening, compared with Barrett's esophagus screening and surveillance. A cost-benefit analysis studied standard endoscopic screening strategies for esophageal cancer in high-risk areas of China. The authors found that, compared with no screening, all screening strategies with varying screening ages, frequencies, and follow-up intervals could save more life-years [57]. A recent study used a decision-analytic Markov model to study the cost-effectiveness of incorporating high resolution microendoscopy into an SCC screening program in China, with results showing that it could be cost-effective [58].

There are no European studies suggesting that endoscopic screening for SCC is either necessary or cost-effective. The low incidence of SCC in the European population and the predominance of public health systems might be some of the main reasons why screening for this condition is not an option even in individuals with risk factors. Interestingly, if screening for SCC in the Western world was extended to Barrett's cancer and gastric cancer combined, by performing a single upper endoscopy at the time of screening colonoscopy, it might be a costeffective method to screen for multiple cancers simultaneously, with an incremental cost-effectiveness ratio comparing favorably with commonly performed screening strategies for other cancers [47].

\section{Barrett's esophagus (BE) and esophageal adenocarcinoma (EAC)}

\section{STATEMENT}

Endoscopic screening for Barrett's esophagus (BE)associated adenocarcinoma should only be considered in high-risk individuals or patients (opportunistic): i.e., in those with long-standing gastroesophageal reflux disease symptoms (i.e., $>5$ years) and multiple risk factors (age $\geq 50$ years, white race, male sex, obesity, first-degree relative with $\mathrm{BE}$ or esophageal adenocarcinoma).

\section{Epidemiology: BE and EAC}

Esophageal cancer has a poor 5-year survival of less than 15\% $[59,60]$. Moreover, there has been a striking increase in the incidence of EAC and associated death in most Western countries over the past 30 years $[61,62]$. BE is a premalignant condition for the development of EAC, characterized by the replacement of the normal squamous epithelium above the gastroesophageal junction with columnar epithelium $[63,64]$. The prevalence of $\mathrm{BE}$ in the general population has been estimated to be $1 \%$ to $2 \%$ $[65,66]$. The annual risk of BE converting to EAC, after excluding the cases diagnosed during the first year, is $0.12 \%$ to $0.50 \%$ $[67,68]$. Because of this pre-existing condition, EAC can potentially be prevented by screening for this precursor lesion.

\section{Target population: BE and EAC}

Screening for BE or EAC by endoscopic and nonendoscopic methods is not recommended for the general population, because of the relatively low risk. The screening population needs to be enriched by high-risk individuals for screening to be costeffective [70].

Epidemiological studies have identified risk factors for BE and EAC. The main risk factors for $B E$ are gastroesophageal reflux disease (GERD), obesity, male sex, older age, and cigarette smoking [62]. GERD is the strongest established risk factor for $B E$ and $E A C$, with symptoms that have been present for 10 years being especially associated with development of EAC $[61,70]$. $B E$ is more common in men than in women, and among patients with $B E$ there is a 2-3 times higher transformation to EAC in men than women [61]. BE becomes more common with age, and the risk becomes substantial in men older than 60 years who have GERD symptoms [71]. The presence of intestinal metaplasia (i.e., goblet cells) is a risk factor for evolution to neoplasia [63], and is a requirement for fulfilling the ESCE definition of BE. Other risk factors for conversion from BE to $E A C$ are the presence of dysplasia or long-segment BE [63].

\section{Screening age: BE and EAC}

In view of the abovementioned established risk factors, ESGE recommends endoscopic screening after the age of 50-60 years (according to local availability) for patients who have chronic GERD symptoms for more than 5-10 years [72]. The reason why screening high-risk individuals might be beneficial lies in the epidemiological findings that the majority of esophageal cancers are detected at an advanced stage while patients under surveillance for $\mathrm{BE}$ have detection at an earlier stage with better outcomes in comparison to patients who were not under surveillance. Therefore, once the diagnosis of $B E$ is confirmed, patients should be surveilled according to the existing guidelines until the age of 75, if no dysplasia is found, as reported in the ESGE Position Statement.

\section{Role of endoscopy: BE and EAC}

Although endoscopy might be the gold standard investigation, it is invasive and requires staff and expensive equipment [73]. Alternatively, ultrathin nasal endoscopy has been proposed as an alternative, having good accuracy and tolerability for patients. Compared with standard endoscopy, it has been shown to be more cost-effective and to have comparable sensitivity and specificity for the endoscopic diagnosis of BE $[60,74-76]$. Video capsules specifically designed to allow imaging of the esophagus are available. They have a potential advantage over standard endoscopy in terms of tolerability, acceptability, and need for sedation. However, they are quite expensive and studies showed unfavorable diagnostic characteristics with a sensitivity of $60 \%-67 \%$ and specificity of $84 \%-100 \%$ in detection of BE [66].

The Cytosponge is the best studied nonendoscopic screening device for detection of BE. In combination with biomarkers such as p53 it allows risk stratification of patients. A soluble capsule, encasing a small sponge, is swallowed. After entering 
the stomach, the capsule is dissolved and the sponge is recovered by means of an attached string. The cytological sample retrieved by the Cytosponge is then immunostained for trefoil factor 3 (TFF3), a protein encoded by the TFF3 gene [77]. A trial of 1000 patients (BEST2) demonstrated a specificity of $92.4 \%$ and a sensitivity of $80 \%$ for BE, that increased to $87 \%$ for Barrett segments $\geq 3 \mathrm{~cm}$ in circumference. This is an accuracy comparable to those of current screening tests for colorectal and cervical cancer [77]. Although this is a safe method, given the lower sensitivity and specificity of such nonendoscopic screening methods it is not indicated in screening yet. More studies are on the way to further validate the Cytosponge in a primary care setting.

\section{Cost-effectiveness: BE and EAC}

Because of the low risk and incidence of EAC, screening of all individuals is not cost-effective. Even the cost-effectiveness of surveillance of BE is often questioned, since it largely depends on the incidence of neoplasia development in BE [78]. Based upon a cancer incidence of $0.5 \%$ [68], surveillance is costeffective every 5 years for nondysplastic BE and every 3 years for low grade dysplasia in long-segment BE [79]. In regions where the cancer incidence is lower, the usefulness of surveillance is questioned $[60,67]$. One of the problems that compromises the cost-effectiveness of surveillance is the fact that most of the cancers are not detected during surveillance but at the time or within a year of the index endoscopy $[67,68]$. A cost analysis using a microsimulation model for the Cytosponge suggested that in comparison to endoscopy, it had an equal gain in quality-adjusted life-years, but with a higher cost-effectiveness. When combined with endoscopic therapy, it was suggested that the Cytosponge is cost-effective in reducing EAC mortality [80].

\section{Role of digestive endoscopy in high-risk settings: pancreatic cancer}

\section{STATEMENT}

For pancreatic cancer screening, endoscopic ultrasound (EUS) may be used in selected high-risk patients, such as those with a strong family history and/or genetic susceptibility.

\section{Epidemiology: pancreatic cancer}

Pancreatic cancer ranks amongst the most aggressive cancers and has a mortality that nearly equals its incidence [81]. About 100000 new cases of pancreatic cancer and 95000 related deaths have been estimated in Europe in 2018. There have been only small improvements in the 5 -year survival rate over the last two decades, which remains well below $10 \%$. The poor prognosis for the disease is attributed to the aggressive biology, ineffective therapies, and advanced stages at the time of diagnosis $[82,83]$. Thus, detection of precursor lesions or early- stage pancreatic cancer may be an effective approach to improve survival [84].

\section{Target population: pancreatic cancer}

Given the overall low incidence of pancreatic cancer (lifetime risk $1.3 \%$ ), it is not cost-effective to screen the general population [82]. However, selective screening of high-risk individuals is considered beneficial. According to the International Cancer of the Pancreas Screening (CAPS) Consortium, to be a candidate for screening, an individual should have a lifetime risk of $>5 \%$ for pancreatic cancer [85]. These worldwide experts recently published updated consensus criteria for screening individuals based upon their genetic susceptibility or family history. These criteria take into consideration specific genetic mutations and the degree and number of relatives with pancreatic cancer. The CAPS consortium recommends that all patients with Peutz-Jeghers syndrome and all carriers of germline CDKN2A mutation should be screened for pancreatic cancer regardless of family history starting at age 40. Carriers of BRCA2, BRCA1, PALB2, ATM, MLH1, MSH2, or MSH6 germline mutations with at least one affected first-degree relative should be screened as well, starting at age 45 or 50 , or at 10 years younger than the youngest affected blood relative. Individuals who do not have any deleterious mutations, but have at least one affected first-degree relative and one affected second-degree relative on the same side of the family are also considered candidates for high-risk pancreatic cancer screening, starting at age 50 or 55 , or at 10 years younger than the youngest affected blood relative [86].

Recent studies also suggest that patients with new-onset diabetes but without traditional risk factors for diabetes (e. g. metabolic syndrome) are also at increased risk for pancreatic cancer, and could potentially benefit from screening [87-89]. There is still a need for consensus on many issues that are addressed in more detail in the latest updated CAPS consortium guidelines [86].

\section{Role of endoscopy: pancreatic cancer}

The development of a sensitive and specific screening test is crucial for decreasing mortality from pancreatic cancer. Unfortunately, none of the current diagnostic modalities have all the attributes of an effective screening tool, namely, acceptable sensitivity and specificity, low invasiveness, and cost-effectiveness.

EUS is the only endoscopic method that is clinically used for pancreatic cancer screening. EUS has the ability to detect small pancreatic lesions, offers the possibility of tissue sampling, and is the most accepted screening method for early pancreatic cancer detection [85, 90,91].

A number of studies have looked at the efficacy of EUS for the early detection of pancreatic dysplasia and other precursor lesions in high-risk individuals [91-107]. The diagnostic yield of EUS varies widely. According to a recent meta-analysis [108], EUS detected more high-risk lesions (1.07 [95\% Cl 0.05-2.09]) per 100 patient-years) than MRI (0.41 [0.05-0.78]), without reaching statistical difference. Furthermore, EUS detected more cases with chronic pancreatitis. Regarding routine follow- 


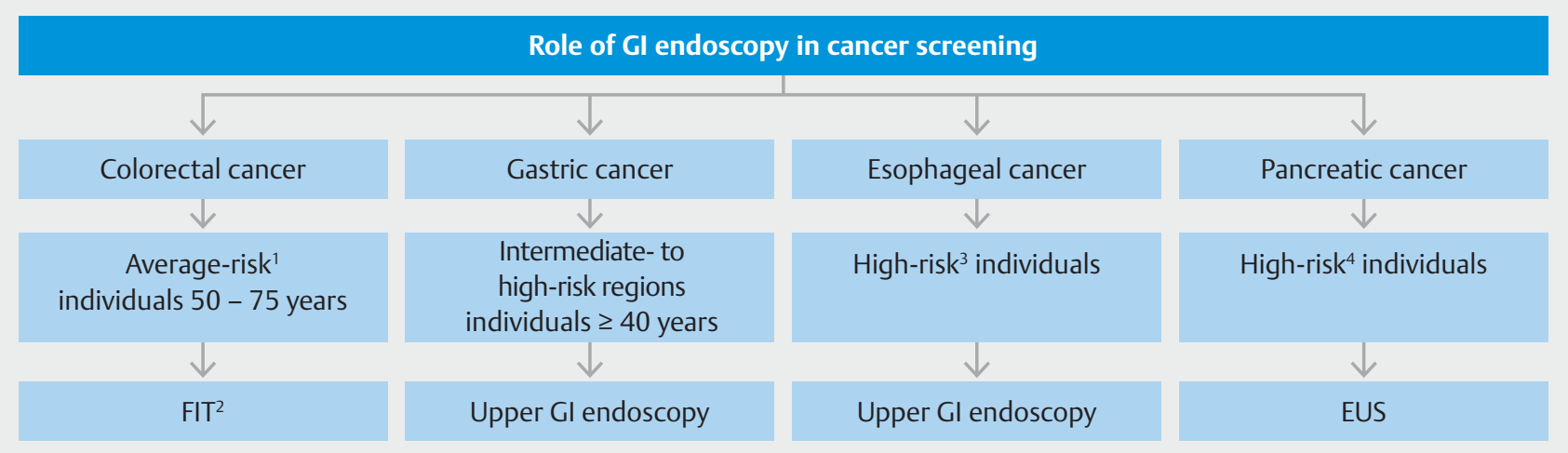

Fig. 1 Role of gastrointestinal (GI) endoscopy in screening for Gl cancer.

${ }^{1}$ For individuals at increased risk of colorectal or gastric cancer see corresponding European Society of Gastrointestinal Endoscopy (ESGE) Guidelines.

${ }^{2}$ Colonoscopy or sigmoidoscopy are also acceptable alternatives.

${ }^{3}$ For squamous cell cancer: personal history of head/neck cancer, achalasia or previous caustic injury. For Barrett's esophagus-associated adenocarcinoma: long-standing gastroesophageal reflux disease (GERD) symptoms (i. e., > 5 years) and multiple risk factors (age $\geq 50$ years, white race, male sex, obesity, first-degree relative with Barrett's esophagus or esophageal adenocarcinoma).

${ }^{4}$ History of familial pancreatic cancer (with an affected first-degree relative) and inherited genetic syndromes.

FIT, fecal immunochemical testing.

up after baseline screening, most published studies used the same imaging tests [85].

Few studies have directly compared the diagnostic yield of imaging tests for high-risk individuals in screening, and most comparisons have not been performed in a blinded, randomized fashion. The prospective CAPS3 study [104] performed blinded comparisons of CT, MRI/MRCP, and EUS for one-time screening. It showed that EUS and MRI are better than $C T$ for the detection of small, predominantly cystic, pancreatic lesions, with good concordance of lesion number, size, and location between EUS and MRI/MRCP. However, it has been shown that EUS was particularly sensitive for the early detection of small solid lesions, while MRI was very sensitive for the detection of (small) cystic lesions [107]. Consequently, the latter authors suggested that within a screening setting, in order to maximize the detection rate of clinically relevant lesions, both EUS and MRI should be considered.

The risk of incorrect diagnosis and overtreatment of lesions identified by EUS remains a significant concern. EUS is an operator-dependent test with only modest interobserver agreement [109]. Furthermore, the role of EUS-guided fine needle aspiration to evaluate pancreatic lesions in high-risk individuals is not well established. It proved to be very accurate (sensitivity $85 \%-89 \%$ and specificity $96 \%-99 \%$ ) in diagnosing solid pancreatic lesions [110]. However, there is a risk of falsepositive cytology results from small lesions that can lead to unnecessary surgery [85]. Moreover, in cystic lesions cytological investigation has low accuracy, and often the volume of cyst fluid aspirated from small cysts is low [111].

The CAPS consortium guidelines recommend screening at baseline with EUS plus MRI/MRCP, along with fasting blood glucose and/or HbA1c. For patients with normal baseline examination findings, the recommendation is to alternate MRI/MRCP plus EUS on an annual basis with fasting blood glucose or HbA1c. No consensus has been reached on if and how to alternate EUS and MRI/MRCP [86].

\section{Cost-effectiveness: pancreatic cancer}

According to CAPS [85], "successful" screening refers to detecting those high-risk lesions for which surgery is beneficial: high grade dysplasia, high grade pancreatic intraepithelial neoplasia (PanIN), or T1N0M0 margin-negative pancreatic cancer.

A recent meta-analysis [108] showed that EUS can detect a large number of pancreatic lesions, but only a small subgroup represent high-risk lesions. Accordingly, there is a risk of overtreatment for pancreatic screening that is magnified by the risks of morbidity and mortality $(\sim 1 \%-2 \%)$ of pancreatic surgery. However, perioperative mortality has significantly declined from $15 \%$ in the 1970 s, to $4 \%$ in modern series, and to less than $2 \%$ in high-volume centers [112-114].

Another approach for assessment of the benefits of pancreatic screening would be to consider its impact on the quality of life of the individuals who are at risk for developing cancer. A recent systematic review showed that high-risk individuals have positive psychological outcomes from participating in pancreatic cancer screening programs [115]. Although screening might not always be reassuring, it may improve individuals' quality of life, and this should be an important consideration with regard to pancreatic cancer screening.

Finally, after many years of efforts in screening high-risk individuals for pancreatic cancer, some impact on survival is now beginning to be realized. Recent data indicate that among individuals at high risk for pancreatic adenocarcinoma who were screened with EUS, MRI, and/or CT, most pancreatic cancers detected during surveillance (9/10) were resectable, and $85 \%$ of the patients survived for at least 3 years [116]. 
Given the evolving nature of this field, screening for pancreatic cancer should preferably be performed within a research setting, or at referral centers with dedicated EUS experts with clinical expertise and interest in this field, in the context of a multidisciplinary high-risk screening program.

\section{Conclusions}

The impact of GI endoscopy on GI cancer prevention is substantial, and it is strictly related to three main variables, namely the absolute burden of each cancer according to country-specific disease incidence, the risk attributable to the target population, and the expected efficacy of screening prevention. Consequently, we stratified the opportunity of endoscopic screening into two main categories, as applied to average-risk individuals or only to high-risk individuals ( $\triangleright$ Fig. 1 ).

When the high accuracy of endoscopy-related GI cancer prevention is coupled with the availability of nonsurgical endoscopic treatment of precancerous and early-invasive lesions, a unique opportunity is presented to eradicate $\mathrm{Gl}$ cancer in an acceptable and efficient manner.

\section{Acknowledgments}

R. Bisschops is supported by a grant from the Research Foundation Flanders (FWO).

\section{Competing interests}

R. Bisschops has received research grants from Pentax, Fujifilm, Cook, and Medtronic; he has been/is on advisory boards for Pentax, Fujifilm, Cook, Boston Scientific, Medtronic, and CDX Diagnostic; he has been a speaker for Pentax, Fujifilm, Medtronic, and Medivators; he has received organizational support for educational events from Pentax, and organizational and financial support for educational events from Cook, Boston Scientific, Medtronic, Erbe, and Olympus. E. Dekker has received honoraria from Fujifilm, Olympus, Tillots, and GI Supply, and speaker's fees from CPP-FAP and Olympus; she is a Co-Editor of Endoscopy journal. H. Messmann has received, in the past 3 years: grants from the Falk Foundation, Olympus, Roche, Bayer, MSD, Novartis, and Amgen; honoraria from the Falk Foundation, Olympus, and Covidien; consultation fees from Lumendi, Boston Scientific, Erbe, and Olympus; and research support from Olympus (ESD-Register). A. Saftoiu has received lecture fees from Pentax Medical Singapore (20162019); his department at Ponderas Academic Hospital received 3000 FIT tests (pilot colorectal cancer screening) from Johnson and Johnson (2019-2020). P.D. Siersema is receiving research support from Pentax (2018-2021) and Yakult (2017-2020); his department is receiving research support from The eNose Company (2019-2021); he is Editor-in-Chief of Endoscopy journal. T. Ponchon is providing consultancy to Olympus, Norgine, Ipsen, and Alfa Sigma (from 1 January 2019 to present); his department is receiving research funding from Boston Scientific. J.E. van Hooft has received lecture fees from Medtronics (2014-2015, 2018) and Cook Medical (2019), and consultancy fees from Boston Scientific (2014-2017); her department has received research grants from Cook Medical (2014-2018) and Abbott (2014-2017). G. Antonelli, M. Areia, M.S. Bhutani, E. Bories, R. Capocaccia, I.M. Cazacu, P.H. Deprez, M. Dinis-Ribeiro, C. Hassan, S.P. Pereira, and C. Senore declare no competing interests.

\section{References}

[1] Survival of Cancer Patients in Europe. Eurocare 5 database. Accessed: 16 Sept 2019, Available at: https://w3.iss.it/site/EU5Results/

[2] van Leerdam ME, Roos VH, van Hooft JE et al. Endoscopic management of Lynch syndrome and of familial risk of colorectal cancer: European Society of Gastrointestinal Endoscopy (ESGE) Guideline. Endoscopy 2019; 51: 1082-1093

[3] Pimentel-Nunes P, Libânio D, Marcos-Pinto R et al. Management of epithelial precancerous conditions and lesions in the stomach (MAPS II): European Society of Gastrointestinal Endoscopy (ESGE), European Helicobacter and Microbiota Study Group (EHMSG), European Society of Pathology (ESP), and Sociedade Portuguesa de Endoscopia Digestiva (SPED) guideline update 2019. Endoscopy 2019; 51: 365-388

[4] ECIS. ECIS - European Cancer Information System. Accessed: 15 Sept 2019, Available from: https://ecis.jrc.ec.europa.eu

[5] RARECARENet Information Network on Rare Cancers. Accessed: 16 Sept 2019, Available from: http://rarecarenet.eu/index.php

[6] IARC. Available at: https://gco.iarc.fr/today/data/factsheets/cancers/10_8_9-Colorectum-fact-sheet.pdf

[7] Vuik FE, Nieuwenburg SA, Bardou M et al. Increasing incidence of colorectal cancer in young adults in Europe over the last 25 years. Gut 2019; 68: 1820-1826

[8] Brenner H, Stock C, Hoffmeister M. Effect of screening sigmoidoscopy and screening colonoscopy on colorectal cancer incidence and mortality: systematic review and meta-analysis of randomised controlled trials and observational studies. BMJ 2014; 348: g2467

[9] Holme $\varnothing$, Bretthauer M, Fretheim A et al. Flexible sigmoidoscopy versus faecal occult blood testing for colorectal cancer screening in asymptomatic individuals. Cochrane Database Syst Rev 2013: CD009259

[10] Hassan C, Giorgi Rossi P, Camilloni L et al. Meta-analysis: adherence to colorectal cancer screening and the detection rate for advanced neoplasia, according to the type of screening test. Aliment Pharmacol Ther 2012; 36: 929-940

[11] Atkin WS, Edwards R, Kralj-Hans I et al. Once-only flexible sigmoidoscopy screening in prevention of colorectal cancer: a multicentre randomised controlled trial. Lancet 2010; 375: 1624-1633

[12] Segnan N, Armaroli P, Bonelli L et al. Once-only sigmoidoscopy in colorectal cancer screening: follow-up findings of the Italian Randomized Controlled Trial-SCORE. J Natl Cancer Inst 2011; 103: 1310-1322

[13] Brenner H, Arndt V, Stürmer T et al. Long-lasting reduction of risk of colorectal cancer following screening endoscopy. Br J Cancer 2001; 85: 972-976

[14] Singal AG, Gupta S, Skinner CS et al. Effect of colonoscopy outreach vs fecal immunochemical test outreach on colorectal cancer screening completion: A randomized clinical trial. JAMA 2017; 318: 806-815

[15] Lauby-Secretan B, Vilahur N, Bianchini F et al. International Agency for Research on Cancer Handbook Working Group. The IARC perspective on colorectal cancer screening. N Engl J Med 2018; 378 : 1734-1740

[16] Kaminski MF, Thomas-Gibson S, Bugajski M et al. Performance measures for lower gastrointestinal endoscopy: a European Society of Gastrointestinal Endoscopy (ESGE) Quality Improvement Initiative. Endoscopy 2017; 49: 378-397

[17] Chen C, Läcke E, Stock C et al. Colonoscopy and sigmoidoscopy use among older adults in different countries: A systematic review. Prev Med 2017; 103: 33-42

[18] Senore C, Inadomi ], Segnan N et al. Optimising colorectal cancer screening acceptance: a review. Gut 2015; 64: 1158-1177 
[19] Basu P, Ponti A, Anttila A et al. Status of implementation and organization of cancer screening in The European Union Member States Summary results from the second European screening report. Int ] Cancer 2018; 142: 44-56

[20] Eisinger F, Cals L, Calazel-Benque A et al. Impact of organised programs on colorectal cancer screening. BMC Cancer 2008; 8: 104

[21] van Turenhout ST, Terhaar sive Droste JS, Meijer GA et al. Anticipating implementation of colorectal cancer screening in The Netherlands: a nationwide survey on endoscopic supply and demand. BMC Cancer 2012; 12: 46

[22] Parente F, Marino B, Ardizzoia A et al. Impact of a population-based colorectal cancer screening program on local health services demand in Italy: a 7-year survey in a northern province. Am J Gastroenterol 2011; 106: 1986-1993

[23] Zorzi M, Senore C, Turrin A et al. Appropriateness of endoscopic surveillance recommendations in organised colorectal cancer screening programmes based on the faecal immunochemical test. Gut 2016; 65: 1822-1828

[24] Schreuders EH, Ruco A, Rabeneck L et al. Colorectal cancer screening: a global overview of existing programmes. Gut 2015; 64: 16371649

[25] Senore C, Basu P, Anttila A et al. Performance of colorectal cancer screening in the European Union Member States: data from the second European screening report. Gut 2019; 68: 1232-1244

[26] Lansdorp-Vogelaar I, Knudsen A, Brenner H. Cost-effectiveness of colorectal cancer screening - an overview. Best Pract Res Clin Gastroenterol 2010; 24: 439-449

[27] Torre LA, Bray F, Siegel RL et al. Global cancer statistics, 2012. CA Cancer J Clin 2015; 65: 87-108

[28] Zhang X, Li M, Chen S et al. Endoscopic screening in Asian countries is associated with reduced gastric cancer mortality: A meta-analysis and systematic review. Gastroenterology 2018; 155: 347-354.e9

[29] Correa P. Human gastric carcinogenesis: a multistep and multifactorial process - First American Cancer Society Award Lecture on Cancer Epidemiology and Prevention. Cancer Res 1992; 52: 67356740

[30] Hamashima C. Systematic Review Group and Guideline Development Group for Gastric Cancer Screening Guidelines. Update version of the Japanese Guidelines for Gastric Cancer Screening. Ipn J Clin Oncol 2018; 48: 673-683

[31] Kim B], Heo C, Kim BK et al. Effectiveness of gastric cancer screening programs in South Korea: organized vs opportunistic models. World J Gastroenterol 2013; 19: 736-741

[32] Pimentel-Nunes P, Dinis-Ribeiro M, Ponchon T et al. Endoscopic submucosal dissection: European Society of Gastrointestinal Endoscopy (ESGE) Guideline. Endoscopy 2015; 47: 829-854

[33] Ono H, Yao K, Fujishiro M et al. Guidelines for endoscopic submucosal dissection and endoscopic mucosal resection for early gastric cancer. Dig Endosc 2016; 28: 3-15

[34] Dinis-Ribeiro M, Yamaki G, Miki K et al. Meta-analysis on the validity of pepsinogen test for gastric carcinoma, dysplasia or chronic atrophic gastritis screening. J Med Screen 2004; 11: 141-147

[35] Lomba-Viana R, Dinis-Ribeiro M, Fonseca F et al. Serum pepsinogen test for early detection of gastric cancer in a European country. Eur J Gastroenterol Hepatol 2012; 24: 37-41

[36] Huang Y, Yu J, Kang W et al. Significance of serum pepsinogens as a biomarker for gastric cancer and atrophic gastritis screening: A systematic review and meta-analysis. PLoS One 2015; 10: e0142080 Accessed: 24 Oct 2019, Available at: https://www.ncbi.nlm.nih.gov/ pmc/articles/PMC4640555

[37] Kim GH, Liang PS, Bang S] et al. Screening and surveillance for gastric cancer in the United States: Is it needed? Gastrointest Endosc 2016; 84: $18-28$
[38] Areia M, Spaander MC, Kuipers E] et al. Endoscopic screening for gastric cancer: A cost-utility analysis for countries with an intermediate gastric cancer risk. United Eur Gastroenterol J 2018; 6: 192 202

[39] Tashiro A, Sano M, Kinameri K et al. Comparing mass screening techniques for gastric cancer in Japan. World J Gastroenterol 2006; 12: $4873-4874$

[40] Zhou L, Guan P, Sun L et al. Health economic assessment for screening of gastric cancer in a high risk population in northeastern China. Chin J Cancer Res 2011; 23: 21-24

[41] Lee H-Y, Park E-C, Jun J-K et al. Comparing upper gastrointestinal $\mathrm{X}$-ray and endoscopy for gastric cancer diagnosis in Korea. World J Gastroenterol 2010; 16: 245-250

[42] Chang H-S, Park E-C, Chung W et al. Comparing endoscopy and upper gastrointestinal $X$-ray for gastric cancer screening in South Korea: a cost-utility analysis. Asian Pac J Cancer Prev 2012; 13: 27212728

[43] Areia M, Carvalho R, Cadime AT et al. Screening for gastric cancer and surveillance of premalignant lesions: a systematic review of cost-effectiveness studies. Helicobacter 2013; 18: 325-337

[44] Cho E, Kang MH, Choi KS et al. Cost-effectiveness outcomes of the national gastric cancer screening program in South Korea. Asian Pac J Cancer Prev 2013; 14: 2533-2540

[45] Zhou HJ, Dan YY, Naidoo N et al. A cost-effectiveness analysis evaluating endoscopic surveillance for gastric cancer for populations with low to intermediate risk. PloS One 2013; 8: e83959

[46] Wu JT, Zhou J, Naidoo $\mathrm{N}$ et al. Determining the cost-effectiveness of endoscopic surveillance for gastric cancer in patients with precancerous lesions. Asia Pac J Clin Oncol 2016; 12: 359-368

[47] Gupta N, Bansal A, Wani SB et al. Endoscopy for upper GI cancer screening in the general population: a cost-utility analysis. Gastrointest Endosc 2011; 74: 610-624.e2

[48] Yeh JM, Hur C, Ward Z et al. Gastric adenocarcinoma screening and prevention in the era of new biomarker and endoscopic technologies: a cost-effectiveness analysis. Gut 2016; 65: 563-574

[49] Oesophageal cancer statistics. World Cancer Research Fund 2018 Accessed: 24 Oct 2019, Available at: https://www.wcrf.org/dietandcancer/cancer-trends/oesophageal-cancer-statistics

[50] Rossini AR, Hashimoto CL, Iriya K et al. Dietary habits, ethanol and tobacco consumption as predictive factors in the development of esophageal carcinoma in patients with head and neck neoplasms. Dis Esophagus 2008; 21: 316-321

[51] Muto M, Hironaka S, Nakane M et al. Association of multiple Lugolvoiding lesions with synchronous and metachronous esophageal squamous cell carcinoma in patients with head and neck cancer. Gastrointest Endosc 2002; 56: 517-521

[52] Zendehdel K, Nyrén O, Edberg A et al. Risk of esophageal adenocarcinoma in achalasia patients, a retrospective cohort study in Sweden. Am J Gastroenterol 2011; 106: 57-61

[53] Blaydon DC, Etheridge SL, Risk JM et al. RHBDF2 mutations are associated with tylosis, a familial esophageal cancer syndrome. Am J Hum Genet 2012; 90: 340-346

[54] Ravi K, Geno DM, Katzka DA. Esophageal cancer screening in achalasia: is there a consensus? Dis Esophagus 2015; 28: 299-304

[55] Smyth EC, Lagergren J, Fitzgerald RC et al. Oesophageal cancer. Nat Rev Dis Primer 2017; 3: 17048

[56] Pan Q-J, Roth MJ, Guo H-Q et al. Cytologic detection of esophageal squamous cell carcinoma and its precursor lesions using balloon samplers and liquid-based cytology in asymptomatic adults in Llinxian, China. Acta Cytol 2008; 52: 14-23

[57] Yang J, Wei W-Q, Niu J et al. Cost-benefit analysis of esophageal cancer endoscopic screening in high-risk areas of China. World J Gastroenterol 2012; 18: 2493-2501 
[58] Hur C, Choi SE, Kong CY et al. High-resolution microendoscopy for esophageal cancer screening in China: A cost-effectiveness analysis. World J Gastroenterol 2015; 21: 5513-5523

[59] Herszényi L, Tulassay Z. Epidemiology of gastrointestinal and liver tumors. Eur Rev Med Pharmacol Sci 2010; 14: 249-258

[60] Blot W], Devesa SS, Kneller RW et al. Rising incidence of adenocarcinoma of the esophagus and gastric cardia. JAMA 1991; 265: 12871289

[61] Edgren G, Adami H-O, Weiderpass E et al. A global assessment of the oesophageal adenocarcinoma epidemic. Gut 2013; 62: 1406-1414

[62] Thrift AP. The epidemic of oesophageal carcinoma: Where are we now? Cancer Epidemiol 2016; 41: 88-95

[63] Fitzgerald RC, di Pietro M, Ragunath K et al. British Society of Gastroenterology guidelines on the diagnosis and management of Barrett's oesophagus. Gut 2014; 63: 7-42

[64] Bennett C, Moayyedi P, Corley DA et al. BOB CAT: A large-scale review and Delphi consensus for management of Barrett's esophagus with no dysplasia, indefinite for, or low-grade dysplasia. Am J Gastroenterol 2015; 110: 662-682

[65] Ronkainen J, Aro P, Storskrubb T et al. Prevalence of Barrett's esophagus in the general population: an endoscopic study. Gastroenterology 2005; 129: 1825-1831

[66] di Pietro M, Chan D, Fitzgerald RC et al. Screening for Barrett's esophagus. Gastroenterology 2015; 148: 912-923

[67] Hvid-Jensen F, Pedersen L, Drewes AM et al. Incidence of adenocarcinoma among patients with Barrett's esophagus. N Engl J Med 2011; 365: 1375-1383

[68] Sharma P, Falk GW, Weston AP et al. Dysplasia and cancer in a large multicenter cohort of patients with Barrett's esophagus. Clin Gastroenterol Hepatol 2006; 4: 566-572

[69] di Pietro M, Canto MI, Fitzgerald RC. Endoscopic management of early adenocarcinoma and squamous cell carcinoma of the esophagus: Screening, diagnosis, and therapy. Gastroenterology 2018; 154: 421-436

[70] Lagergren J, Bergström R, Lindgren A et al. Symptomatic gastroesophageal reflux as a risk factor for esophageal adenocarcinoma. N Engl J Med 1999; 340: 825-831

[71] Rubenstein JH, Scheiman JM, Sadeghi S et al. Esophageal adenocarcinoma incidence in individuals with gastroesophageal reflux: synthesis and estimates from population studies. Am J Gastroenterol 2011; 106: 254-260

[72] Weusten B, Bisschops R, Coron E et al. Endoscopic management of Barrett's esophagus: European Society of Gastrointestinal Endoscopy (ESGE) Position Statement. Endoscopy 2017; 49: 191-198

[73] Sharma P, Dent J, Armstrong D et al. The development and validation of an endoscopic grading system for Barrett's esophagus: the Prague C \& M criteria. Gastroenterology 2006; 131: 1392-1399

[74] Kadri S, Lao-Sirieix P, Fitzgerald RC. Developing a nonendoscopic screening test for Barrett's esophagus. Biomark Med 2011; 5: 397404

[75] Trevisani L, Cifalà V, Sartori S et al. Unsedated ultrathin upper endoscopy is better than conventional endoscopy in routine outpatient gastroenterology practice: a randomized trial. World J Gastroenterol 2007; 13: 906-911

[76] Shariff MK, Bird-Lieberman EL, O’Donovan M et al. Randomized crossover study comparing efficacy of transnasal endoscopy with that of standard endoscopy to detect Barrett's esophagus. Gastrointest Endosc 2012; 75: 954-961

[77] Ross-Innes CS, Debiram-Beecham I, O’Donovan M et al. Evaluation of a minimally invasive cell sampling device coupled with assessment of trefoil factor 3 expression for diagnosing Barrett's esophagus: a multi-center case-control study. PLoS Med 2015; 12 : e1001780
[78] Sharma P. Clinical practice. Barrett's esophagus. N Engl J Med 2009; 361: 2548-2556

[79] Kastelein F, van Olphen S, Steyerberg EW et al. Surveillance in patients with long-segment Barrett's oesophagus: a cost-effectiveness analysis. Gut 2015; 64: 864-871

[80] Benaglia T, Sharples LD, Fitzgerald RC et al. Health benefits and cost effectiveness of endoscopic and nonendoscopic cytosponge screening for Barrett's esophagus. Gastroenterology 2013; 144: 6273.e6

[81] Xiao AY, Tan MLY, Wu LM et al. Global incidence and mortality of pancreatic diseases: a systematic review, meta-analysis, and metaregression of population-based cohort studies. Lancet Gastroenterol Hepatol 2016; 1: 45-55

[82] Zhang Q, Zeng L, Chen Y et al. Pancreatic cancer epidemiology, detection, and management. Gastroenterol Res Pract 2016; 2016: 8962321

[83] Oberstein PE, Olive KP. Pancreatic cancer: why is it so hard to treat? Ther Adv Gastroenterol 2013; 6: 321-337

[84] McAllister F, Montiel MF, Uberoi GS et al. Current status and future directions for screening patients at high risk for pancreatic cancer. Gastroenterol Hepatol 2017; 13: 268-375

[85] Canto MI, Harinck F, Hruban RH et al. International Cancer of the Pancreas Screening (CAPS) Consortium summit on the management of patients with increased risk for familial pancreatic cancer. Gut 2013; 62: 339-347

[86] Goggins M, Overbeek KA, Brand R et al. International Cancer of the Pancreas Screening (CAPS) consortium. Management of patients with increased risk for familial pancreatic cancer: updated recommendations from the International Cancer of the Pancreas Screening (CAPS) Consortium. Gut 2020; 69: 7-17

[87] Ogawa $\mathrm{Y}$, Tanaka M, Inoue $\mathrm{K}$ et al. A prospective pancreatographic study of the prevalence of pancreatic carcinoma in patients with diabetes mellitus. Cancer 2002; 94: 2344-2349

[88] Illés D, Terzin V, Holzinger $G$ et al. New-onset type 2 diabetes mellitus - A high-risk group suitable for the screening of pancreatic cancer? Pancreatology 2016; 16: 266-271

[89] Molina-Montes E, Gómez-Rubio P, Malats N. Type 2 diabetes mellitus and pancreatic cancer risk. An independent etiological relation? Pancreatology 2018; 18: S7

[90] Bhutani MS, Koduru P, Joshi V et al. The role of endoscopic ultrasound in pancreatic cancer screening. Endosc Ultrasound 2016; 5: 8-16

[91] DaVee T, Coronel E, Papafragkakis C et al. Pancreatic cancer screening in high-risk individuals with germline genetic mutations. Gastrointest Endosc 2018; 87: 1443-1450

[92] Brentnall TA, Bronner MP, Byrd DR et al. Early diagnosis and treatment of pancreatic dysplasia in patients with a family history of pancreatic cancer. Ann Intern Med 1999; 131: 247-255

[93] Poley JW, Kluijt I, Gouma DJ et al. The yield of first-time endoscopic ultrasound in screening individuals at a high risk of developing pancreatic cancer. Am J Gastroenterol 2009; 104: 2175-2181

[94] Langer P, Kann PH, Fendrich V et al. Five years of prospective screening of high-risk individuals from families with familial pancreatic cancer. Gut 2009; 58: 1410-1418

[95] Sud A, Wham D, Catalano M et al. Promising outcomes of screening for pancreatic cancer by genetic testing and endoscopic ultrasound. Pancreas 2014; 43: 458-461

[96] Zubarik R, Gordon SR, Lidofsky SD et al. Screening for pancreatic cancer in a high-risk population with serum CA 19-9 and targeted EUS: a feasibility study. Gastrointest Endosc 2011; 74: 87-95

[97] Ludwig E, Olson SH, Bayuga $S$ et al. Feasibility and yield of screening in relatives from familial pancreatic cancer families. Am J Gastroenterol 2011; 106: 946-954 
[98] Verna EC, Hwang C, Stevens PD et al. Pancreatic cancer screening in a prospective cohort of high-risk patients: a comprehensive strategy of imaging and genetics. Clin Cancer Res 2010; 16: 5028-5037

[99] Mocci E, Guillen-Ponce C, Earl J et al. PanGen-Fam: Spanish registry of hereditary pancreatic cancer. Eur J Cancer 2015; 51: 1911-1917

[100] Al-Sukhni W, Borgida A, Rothenmund $\mathrm{H}$ et al. Screening for pancreatic cancer in a high-risk cohort: an eight-year experience. J Gastrointest Surg 2012; 16: 771-783

[101] Lucas AL, Frado LE, Hwang $C$ et al. BRCA1 and BRCA2 germline mutations are frequently demonstrated in both high-risk pancreatic cancer screening and pancreatic cancer cohorts. Cancer 2014; 120 : 1960-1967

[102] Canto MI, Goggins M, Hruban RH et al. Screening for early pancreatic neoplasia in high-risk individuals: a prospective controlled study. Clin Gastroenterol Hepatol 2006; 4: 766-781; quiz 665

[103] Canto MI, Goggins M, Yeo C] et al. Screening for pancreatic neoplasia in high-risk individuals: an EUS-based approach. Clin Gastroenterol Hepatol 2004; 2: 606-621

[104] Canto MI, Hruban RH, Fishman EK et al. Frequent detection of pancreatic lesions in asymptomatic high-risk individuals. Gastroenterology 2012; 142: 796-804; quiz e14-15

[105] Shin EJ, Topazian M, Goggins MG et al. Linear-array EUS improves detection of pancreatic lesions in high-risk individuals: a randomized tandem study. Gastrointest Endosc 2015; 82: 812-818

[106] Gangi A, Malafa M, Klapman J. Endoscopic ultrasound-based pancreatic cancer screening of high-risk individuals: A prospective observational trial. Pancreas 2018; 47: 586-591

[107] Harinck F, Konings IC, Kluijt I et al. A multicentre comparative prospective blinded analysis of EUS and MRI for screening of pancreatic cancer in high-risk individuals. Gut 2016; 65: 1505-1513
[108] Corral JE, Mareth KF, Riegert-Johnson DL et al. Diagnostic yield from screening asymptomatic individuals at high risk for pancreatic cancer: A meta-analysis of cohort studies. Clin Gastroenterol Hepatol 2019; 17: 41-53

[109] Topazian M, Enders F, Kimmey M et al. Interobserver agreement for EUS findings in familial pancreatic-cancer kindreds. Gastrointest Endosc 2007; 66: 62-67

[110] Cazacu IM, Luzuriaga Chavez AA, Saftoiu A et al. A quarter century of EUS-FNA: Progress, milestones, and future directions. Endosc Ultrasound 2018; 7: 141-160

[111] Brugge WR, Lewandrowski K, Lee-Lewandrowski E et al. Diagnosis of pancreatic cystic neoplasms: a report of the cooperative pancreatic cyst study. Gastroenterology 2004; 126: 1330-1336

[112] Yeo C], Cameron JL, Sohn TA et al. Six hundred fifty consecutive pancreaticoduodenectomies in the 1990s: pathology, complications, and outcomes. Ann Surg 1997; 226: 248-257; discussion 257-260

[113] Balcom JH, Rattner DW, Warshaw AL et al. Ten-year experience with 733 pancreatic resections: changing indications, older patients, and decreasing length of hospitalization. Arch Surg 2001; 136: 391-398

[114] Stauffer JA, Onkendi EO, Wallace MB et al. Standardization and streamlining of a pancreas surgery practice improves outcomes and resource utilization: A single institution's 20-year experience. Am J Surg 2017; 214: 450-455

[115] Cazacu IM, Luzuriaga Chavez AA et al. Psychological impact of pancreatic cancer screening by EUS or magnetic resonance imaging in high-risk individuals: A systematic review. Endosc Ultrasound 2019; 8: $17-24$

[116] Canto MI, Almario JA, Schulick RD et al. Risk of neoplastic progression in individuals at high risk for pancreatic cancer undergoing long-term surveillance. Gastroenterology 2018; 155: 740-751 